\title{
PROCEEDINGS OF THE FIRST MEETING OF THE CENTRAL SOCIETY FOR CLINICAL RESEARCH HELD IN CHICAGO, ILL., NOVEMBER 23, 1928
}

The Influence of Respiration on Venous Pressure. By O. O. MEYER, M.D. (by invitation), and WM. S. MmDleton, M.D., Madison, Wis.

Movement of blood into the thorax is favored by the increased negativity of intrapleural pressure on inspiration. Accordingly venous pressure in the accessible peripheral veins of the forearm falls on inspiration. With the initiation of expiration and the coincident fall of intrapleural negative pressure, peripheral venous pressure increases. Under ordinary circumstances in the normal individual these changes are too insignificant to warrant clinical attention. However, the theoretical considerations stated develop a practical importance when disturbances of respiratory rhythm occur, as in the induction of anesthesia and CheyneStokes respiration. The physical exertion, respiratory arrest and probable carbon dioxide increase of the induction stage of general anesthesia lead to sharp rises of the venous pressure. The period of hyperpnoea of Cheyne-Stokes respiration invariably induces a fall of venous pressure whereas an increase attends the apneic phase.

Led by these observations to seek a practical application as a dynamic test for right ventricular integrity, a series of studies was pursued relative to the venous pressure response to the Valsalva and to the Mueller tests. Briefly stated the results confirm the concensus of opinion as to the rise of venous pressure with the Valsalva experiment, but refute the belief that the Mueller test leads to a reduction of venous pressure. In both instances in normal subjects a rise is recorded whose level apparently bears a direct relation to the duration of the experiment and to the energy of the resisted respiratory effort. Obviously the results from the Valsalva experiment have a definite bearing on the circulatory fatalities in child-birth and on the so-called "bed pan" deaths in cardiac cases.

Further developments of this attack led to the application of the Valsalva experiment to the various stages of cardiac incompetency. It is believed that more prompt and higher early elevations of the venous pressure may be anticipated in event of right ventricular weakness; but the resisted expiratory effort can not be maintained under this circumstance. The ultimate level of venous pressure in decompensated individuals under the conditions of the Valsalva experiment therefore does not approach that noted in normal controls. Hence a prognostic value attaches to the study of the venous pressure reaction of such cases to the Valsalva experiment. 
The Cardiodynamic Effects of Stenosis of the Aorta on the Pulmonary Circuit and Right Heart. By Louis N. Katz, M.D., and Mortimer L. Siegel, M.D. (by invitation), Cleveland, Ohio.

An attempt was made in the present investigation to determine whether or not the left ventricle can counteract the effects of an impediment to the onward flow of blood and prevent back pressure effects in the pulmonary artery and right heart. A marked constriction was applied at the root of the aorta and its effect on the pressure pulses in the left auricle, pulmonary artery, and right ventricle was followed in optically recorded curves.

Obstruction of the coronary return flow to the right heart had little or no effect on the curves showing that changes produced were due to an increased coronary flow.

Our results indicate that stenosis of the root of the aorta leads to a damming back of blood in the pulmonary artery and right ventricle sufficient to raise the pressure within them. The left ventricle, left auricle, and pulmonary veins are apparently inadequate to accommodate the excess.

Observations on the Rumpel-Leede Phenomenon. By Johnson McGuire, M.D. (by invitation), Cincinnati, Ohio.

The occurrence of petechial cutaneous hemorrhages following artificially induced venous stasis has been described in various diseases.

Hecht, in 1907, published an account of petechiae observed in children ill with scarlet fever. These hemorrhages developed when loose folds of skin were picked up and held lightly between the fingers.

During an epidemic of scarlet fever, in 1911, Rumpel and Leede noted the appearance of similar hemorrhages following the application of a tourniquet. They came to the conclusion that the test was specific for scarlet fever.

Stephen and later Weissman studied skin hemorrhages in a fairly large series of miscellaneous cases. Using the cuff of an ordinary sphygmomanometer stasis was induced by raising the pressure to $70 \mathrm{mms}$. of $\mathrm{Hg}$ and maintaining this level for five minutes.

Petechial hemorrhages occurred regularly in subacute bacterial endocarditis, frequently in cases of hypertension, occasionally in rheumatic fever, scurvy and acute nephritis, never in tuberculosis, 'staphylococcus infections, pernicious anemia, or diabetes.

Further studies have been made on patients with a variety of diseases and on a number of normal controls.

Description of method. The result in groups of individuals suffering from 25 diseases are recorded in table 1.

The results in $\mathbf{5 0}$ normals in table 2.

An examination of the results in table 1 , shows a markedly positive result with the tourniquet test in all cases of subacute bacterial endocarditis. Inconstant findings occurred in the other diseases. 
In the hypertensive group Weissmann obtained an extremely high percentage of positive reactions, while this series shows a number of negatives with equally high blood pressures. The probable explanation of this discrepancy is the fact that practically every one will give a positive result when the pressure is maintained at $100 \mathrm{~mm}$. for five minutes. To produce what he considered appropriate stasis Weissmann used pressures just below the diastolic levels in the cases of hypertension.

Table 2 shows that approximately one-third of normals gave slightly positive results.

Summary and conclusions. The occurrence of petechiae following venous stasis has been studied in 25 diseases and in a group of normal controls. The results are given in two tables.

In the few cases of subacute bacterial endocarditis examined, the result was uniformly strongly positive and suggests the sign may prove of differential diagnostic value

The frequency of a slightly positive reaction in normals suggests that only markedly positive results be considered significant.

The Effects of Temporary Occlusion of the Coronary Arteries. By Don C. Sutton, M.D. (by invitation), Chicago, Ill.

1. A method for the study of the effect of temporary occlusion of the coronary arteries in unanesthetized dogs has been developed.

2. Temporary occlusion of the coronary arteries in the dog produces pain which is abolished by removal of the left stellate ganglion, by destruction of the perivascular nerves, but not by severance of the vagi.

3. The visceral pericardium is not sensitive to pain.

The Significance of Electrocardiograms Showing Left Axis Deviation with Inverted $T I$ and $U$ pright TIII and $Q R S$ of Normal Duration. By Drew Luten, M.D., and EDward Grove, M.D. (by invitation), St. Louis, Mo.

Experimental evidence (Wilson and Herrmann) indicates that electrocardiograms of this type result from impairment of conductivity in the right limb of the A-V bundle. Two examples of supporting clinical evidence are presented. Our conception of the cause of this defect refers it to coronary disease.

Such records in our files (237 in number) were found to be confined almost exclusively to patients exhibiting either definite or presumptive arterial disease. Heart disease, when it occurred (56 per cent of cases), was almost invariably of types referable, wholly or in part, to coronary involvement.

The higher incidence of such defect in the right limb is readily explained by the coronary distribution to the two divisions of the A-V bundle (Gross).

Emaciation, Anemia, Betany, Chronic Diarrhea and Malabsorption of Fat: A Nutritional Disturbance in Adults which Resembles Celiac Disease and Sprue. By Wrlliam H. Holmes, M.D. (by invitation), Chicago, Ill.

The article deals with a group of six cases in which emaciation, anemia, tetany 
and chronic diarrhea were the dominant symptoms. In certain instances the emaciation amounted to a loss of 50 per cent of the usual body weight. The anemia resembled Addisonian anemia but differed in that there were no signs of postero-lateral sclerosis or of an acidity. Subjective symptoms consisting of tingling and numbness of the extremities were due to tetany and disappeared immediately with restoration of a normal blood calcium. The emaciation and anemia appear to be due to malabsorption of food. Starches and fats are poorly utilized. A high protein diet was well tolerated and did not aggravate the tetany. A markedly dilated and redundant colon was present in all cases. Dietetic control and restoration of normal mineral balance resulted in a cure in two instances and improvement in the remainder.

Chemical Studies of Patients with Non-Tropical Sprue. By Paul StarR, M.D., Chicago, Ill.

This report consists of (1) a detailed account of the results of diet, thyroid, parathyroid and calcium medication on the metabolic rate, weight, blood calcium and stools of a patient with non-tropical sprue observed from May 1926 to November 1928; (2) the chemical data of calcium balance metabolism experiments on a second patient on low fat, high protein diet, normal high fat diet, and normal low fat diet, covering twenty-four three day periods during five months observations; (3) the effect of gastric digestion on the blood calcium in normal patients with a few observations on patients with sprue, consisting of a tabulation of the change in the blood calcium during a fractional meal in twenty instances. The suggestions in regard to the calcium metabolism of sprue to be drawn from these studies are as follows: (1) Chronic latent tetany is present in non-tropical sprue; (2) it may become acute with extreme depression of the blood calcium; (3) negative calcium balance could not be detected on low fat diets; (4) negative calcium balance was found on a high fat diet during diarrhea; (5) treatment calculated to correct this is beneficial; (6) there is an associated depressed basal metabolic rate, and (7) benefit from raising it by thyroid administration; (8) there is a slight but definite depression of the blood calcium during the gastric digestion of a fractional testmeal; (9) this is not dependent upon acid secretion.

Hyperparathyroidism. By Harold Bulger, M.D., Henry H. Dixon, M.D. (by invitation) and David P. BARR, M.D., St. Louis, Mo.

We have studied a patient presenting multiple giant-cell bone tumors, hypotonicity of muscles, bilateral nephrolithiasis, hypercalcemia and abnormal tendency to excrete calcium in the urine and a parathyroid tumor.

The removal of the parathyroid tumor caused severe and almost fatal tetany, hypocalcemia, change in the size of the bone tumors and abnormal tendency to the retention of calcium.

The symptoms of this patient closely resemble many cases which are scattered 
through the literature but which have never been sufficiently defined. It is suggested that these are cases of true hyperparathyroidism.

The Instance of Peptic Ulcer as Determined Post Mortem. By A. J. MrLler, M.D., Louisville, $\mathrm{Ky}$.

Careful examination of the stomach and duodenum in patients over fifteen years of age coming to autopsy at the University Hospital in Omaha, Nebraska, over a period of two years revealed an instance of peptic ulcet of 18.8 per cent. There are 202 cases in the series. Thirty-eight of them had definite evidence of ulceration in the stomach or duodenum; five of these, or 13.1 per cent were diagnosed clinically; thirty-three, or 86.9 per cent, were not recognized. Twenty-one, or 55.2 per cent, were entirely healed lesions, and of these, fourteen, or two-thirds of the healed lesions, were in the duodenum. Thirteen, or 34.3 per cent, were active lesions, and of these, three, or approximately one-third, were in the duodenum. In four, or 10.5 per cent, there were active and healed lesions, all of which were in the stomach.

The instance of peptic ulcer is much greater than commonly believed. Those in the duodenum heal much more frequently than those in the stomach.

A Study of Pulmonary Lesions Among Children and Young Adults. By J. A. MYERS, M.D., Minneapolis, Min.

From the routine examinations of more than six thousand children and young adults, those with definite evidence of pulmonary lesions have been followed over a period of several years. They are grouped according to location of lesions.

Primary tuberculous foci and their relation to the adult form of tuberculosis have been studied.

Different steps in the healing of lesions are presented to show how well pulmonary lesions in childhood often heal. In another group, different steps in the advancement of pulmonary lesions are presented. More than a hundred cases of pulmonary lesions among teen-age girls and boys are included.

Studies of Pulmonary Gangrene. By I. PrLot, M.D. (by invitation), Chicago, Ill. The purpose of these studies is to clarify a clinical condition in which the etiology has not been well understood. Bacteriologic studies in our own cases reveal in most instances fusiform bacilli, spirochetes always with other bacteria particularly the pyogenic cocci. As these organisms appear to be identical with those found in the oral cavity it is evident that the infection is due to invasion of mouth organisms into the lower respiratory tract. For the development of lesions in the patient as well as in the experimental animal certain contributing factors are necessary. In patients aspiration of mouth secretions during anesthesia and foreign bodies are outstanding predisposing causes. In the rabbit material introduced intratracheally as a rule results in no change unless a foreign substance (lipiodal) is added. The Levaditi preparations of the lesions often reveal a 
characteristic distribution of the fusospirochetes. Neosalvarsan in certain cases if given early has a favorable influence but in most instances of the diffuse types of gangrene the outcome is unfavorable.

Studies in Syphilis of the Stomach. By Harry A. Singer, M.D. (by invitation), Chicago, Ill.

According to current medical literature, there is a marked discrepancy between the incidence of syphilis of the stomach in the morgue as compared with its occurrence in the clinic. The chief reason .for this difference is variance in opinion as to what constitutes from the anatomic standpoint gastric lues. Pathologists are inclined to disregard all but those cases in which specific changes are demonstrable. A study of a series of cases from the combined clinical and morphological standpoints indicate that at the time most patients with syphilis of the stomach reach the operating or post-mortem table, the lesions are receding or healing and therefore from the microscopic viewpoint not typical of lues. A gradual transition from the active lesion with its classical luetic picture to the healing and healed lesion can be traced by comparing the histologic slides from a series of resected stomachs.

The Course of Syphilitic Heart Disease. By FREDRICK A. Willius, M.D., Rochester, Min.

This study was undertaken to attempt to determine the course of and the factors influencing the expectation of life in patients suffering from cardiovascular syphilis.

From a large material it was possible to select only one hundred cases in which a definite history of the onset of syphilis was available, and in which the date and cause of death could be accurately determined.

These cases were divided into three groups, the first group comprising those cases presenting clinical evidence of syphilitic aortitis; there were seventeen cases, all males. The second group presenting clinical evidence of syphilitic aortitis associated with aortic insufficiency, comprised fifty-nine males. The third group comprised cases of thoracic aneurism, of which there were tbirty-four, all being males.

The groups are considered separately but in the abstract it seems desirable to present average statistics only.

The average age at which syphilis was contracted was twenty-seven years and the average age at which death occurred from heart disease was fifty-seven years; in other words, thirty years elapsed from the time that the disease was contracted until the patient died of heart disease.

Numerous other points are discussed, viz. the incidence of positive serology of the blood and of the spinal fluid in the various groups, the incidence of associated sypbilitic manifestations, the types of cardiac manifestations and the occurrence of associated electrocardiographic phenomena. 
The Veins of the Suprarenal Glands in Patients with Normal and High Blood Pressure. A Study of the Musculature and Volume in 100 Cases. By EDGAR V. AlleN, M.D. (by invitation) Rochester, Minn.

The suprarenal glands removed at autopsy from 50 cases each with normal and high blood pressure were studied with particular reference to the musculature of the veins and the venous vascularization. About 1400 veins were so studied by sectioning the adrenals at areas $1 \mathrm{~cm}$. apart, staining with Van Gieson connective tissue stain and projecting at magnifications varying from 50 to 200 times. The areas of the lumens of the veins were then calculated by means of a planimeter and the readings corrected statistically.

The results of this study determine the condition of the musculature of the suprarenal veins in the two clinical conditions and give evidence concerning the amount of vascularization by veins. The relation of the condition of the musculature and clinical hypertension is discussed.

Repeated Glucose Tolerance Tests. By Henry J. John, M.D., Cleveland, Ohio. The study of borderline cases of diabetes through repeated glucose tolerance tests over a period of years offers interesting observations as to the evolution or the "keeping in check" of diabetes in patients under different living conditions. It also gives us a definite guide as to the degree of improvement in the patient's condition and thus tells us the degree of liberty we can take with his diet. Or again it shows a retrogression, due to carelessness in diet, infections, mental strains or inadequate treatment. Briefly it opens up a study, which after a sufficient length of time ought to throw some light on the fluctuations of the carbohydrate metabolism under varied conditions and what I consider most important-it ought to tell us just what the status of the borderline group of cases is and through that give us a definite idea as to what is the best way of dealing with this group-in other words it will lead us into preventive medicine in the field of diabetes.

Hyperinsulinism. By Frank N. Allan, M.D. (by invitation), Rochester, Minn. Hyperinsulinism is now recognized as a clinical entity, as a result of the study of a case with a constant tendency to hypoglycemia, previously reported in collaboration with Wilder, Power and Robertson. In this case the cause of the trouble was shown to be overproduction of insulin from a carcinoma originating in the islands of the pancreas. Recently two cases have been under observation, presenting a similar tendency to hypoglycemia, necessitating the ingestion of food or sugar at regular intervals during the day and night to prevent the occurrence of mental confusion, unconsciousness, and convulsions. Hyperinsulinism is suspected, although it cannot be definitely proven. In one case the pancreas appeared normal on surgical exploration, and microscopic examination failed to reveal any abnormality. Removal of a part of the pancreas has diminished but has not completely abolished the tendency to hypoglycemia. The patient no longer requires feeding at night and can remain free of symptoms with only three meals during the day. 
Determination of Glucose in Tissues from Normal Hyper-and Hypoglycemia, and Depancreatized Animals. By William Thalhimer, M.D., and Margaret C. Perry, A.M. (by invitation), Milwaukee, Wis.

The method used for determining glucose in tissue is new. The accuracy of the method was tested by many duplicate determinations, by the recovery of a known amount of glucose added to tissue at various steps of the procedure, and in other ways. Analyses were made which seemed to give accurate determinations of "true" and "apparent" glucose in tissues. Very little variation in the amount of "true" glucose in tissues was found in the different conditions studied.

Blood Urobilin. By M. A. BLANkenhorn, M.D., Cleveland, Ohio.

Normal urobilin content of human blood has not been described. Urobilin is frequently found in the blood under certain pathological conditions in large amounts, and is a constant finding in small amounts in normal urine. It has been considered a threshold substance. By refinement in technique of the Schlesinger fluorescence test, human blood can be shown to have a fairly constant amount of urobilin. The method is described, and the amount as determined from one hundred and twenty-eight normals is reported.

The Untoward Effects of Phenylhydrazin in Certain Cases of Polycythemia Vera.

H. Z. GIFFIN, M.D., and H. M. ConNeR, M.D. (by invitation), Rochester, Minn.

The histories of three patients are presented, in which the use of phenylhydrazin was followed by fatal outcome. In view of the excellent therapeutic results that have been reported in polycythemia vera following the use phenylhydrazin it seems wise to utter this word of caution with respect to its use. Analysis is made of the characteristics of the cases in which the drug should be used with great caution, if at all.

Serum Complement Fixation Tests in Blastomycosis and in Monilia Infections. By O. GarCIA, M.D. (by invitation), St. Louis, Mo.

Agglutination tests for blastomycosis have already been described by a number of workers. A survey of the literature reveals no successful work on complement fixation in blastomycosis.

In a series of experiments both on blastomycosis and monilia infections, it has been possible to develop antigens which can be used for complement fixation tests.

Positive tests have been secured on clinical cases of blastomycosis and also on monilia infections of the mouth, lungs and gastrointestinal tract.

Animals receiving experimental inoculations with these fungi develop definite complement fixing antibodies.

The Intradermal Salt Test in Fever. By Charles Duden, M.D. (by invitation) and DaviD P. BarR, M.D., St. Louis, Mo.

The intradermal salt test has been applied to the study of fever. The disap- 
pearance time of the intradermally injected salt is regularly diminished in bacterial fevers and in fevers caused by foreign protein injection. It is unchanged in fevers of equal degree produced by external heat.

Systemic Histamine-like Reactions in Allergy Due to Cold. A Report of Three Cases. By Bayard T. Horten, M.D. (by invitation) and George E. Brown, M.D., Rochester, Minn.

This report is based on the studies carried out on three subjects, all exhibiting skin disturbances and systemic reactions following exposure to moderate grades of cold. The reactions were so similar in these cases as to constitute a most striking clinical syndrome, which, to our knowledge, has never been reported.

Three cases are reported exhibiting local and general symptoms of cold allergy. The local effects on the skin were: Pallor during the exposure period, followed by redness, swelling, increased local heat of the hands after exposure to cold. Following a latency period of three to four minutes, a characteristic systemic reaction appeared, which was quite characteristic of that produced by histamine. There was a fall in blood pressure, a sharp rise in pulse rate, a tendency to syncope, with transitory recovery in fifteen to thirty minutes. Complete recovery from the local reaction occurred in twelve to twenty-four hours. The experimental work carried out gave evidence of the chemical nature of these reactions, and strongly suggested the release of a histamine-like substance in the skin following exposure to cold which, when carried in the general circulation, produced a reaction characteristic of histamine. These clinical observations are further confirmation of the work of Lewis and associates on the presence of histamine or allied substances in the human skin.

The Relation of the Weather to the Pain of Arthritis. By E. B. RENTSCHLER, M.D. (by invitation), Frances Vanzant, M.D. (by invitation) and L. G. Rowntree, M.D., Rochester, Minn.

It has been found that in an arthritic medical service, the pain of infectious arthritis varies markedly with the changes in the weather. The condition of each patient on the service has been reported every day for a year. On completing rounds the doctors on the arthritic service have made a summary of the statements of the patients and recorded the results. At the same time records have been kept in the laboratory of barometric pressure and temperature inside and outside, humidity, sunshine, the presence or absence of storms and the electrical state.

A remarkable correlation has been found between the pain of arthritis and the presence of a storm. In fact, there were only two or three storms in the entire year not reflected in the pain of the patients with arthritis. There is also a remarkable correlation between the changes in barometric pressure and the pain of arthritis. A positive correlation in more than 70 per cent and some sort of correlation in more than 90 per cent of cases has been observed. The amount of 
sunshine also exercises a marked influence. Certain patients claim a capacity to predict storms by their pain and have been checked, and one has been found to be correct over 95 per cent of the time. In patients who believed that the weather in no way influenced their pain almost as striking a correlation was found at times. This study brings up many considerations which have largely been overlooked by the physician.

Urinary Proteins. By William A. Thomas, M.D. (by invitation), Chicago, Ill. In the production of artificial uremia in dogs, there is obtained a protein in the urine which does not originate in the blood. This protein has been identified by appropriate precipitation tests as coming from viscera and muscle and is definite in its immunological reactions.

Later, this disappears and is replaced by serum protein in large quantities. Demonstration of this protein from several organs has been successful.

Later the process so far breaks down this protein that it is not recognizable as such, but combines with serum protein and is excreted in this combination.

The mechanism of excretion of protein through the kidney damaged or undamaged, is discussed and it is believed that the kidney is not permeable to protein which belongs in the circulation of that species but is excreted only when altered by chemical, osmotic or other factors to such an extent that it is no longer the normal serum protein of that organism and that the liver protein combines with serum protein as a mechanism of detoxication and excretion.

Symptomatic Relapses During Liver-Induced Remissions in Pernicious Anemia. By Cyrus C. Strugrs, M.D., and Raphael Isaacs, M.D. (by invitation), Ann Arbor, Mich.

Many patients with pernicious anemia whose blood has returned to normal while taking liver or an adequate liver fraction, experience, during the months following the development of the remission, certain symptoms which are similar to those of a beginning relapse. These include nausea, loss of appetite, weakness, nervousness, diarrhea or constipation, increase in "numbness" of hands and feet, sore tongue and ease of fatigue. If the liver or liver extract is continued until these symptoms pass away or decrease, a true "hematological" relapse is prevented. Some patients discontinue the liver at this time and the anemia reappears. It is evident that the active liver component acts by supplying a substance, the power to elaborate which has been lost, and is never regained. The treatment is symptomatic and does not influence the fundamental disease process. The periodicity of the relapses and remissions remains a characteristic, even though the blood (and bone marrow) have been restored to normal function. 